\title{
Avaliação da estimativa de fixação de carbono em sistemas agroflorestais na região amazônica
}

\section{Carbon fixation estimated assessment in agroforestry systems in the Amazon region}

\author{
Irna Pinheiro Dias ${ }^{1}$, Beatriz Cordeiro Costa ${ }^{2}$, José Felipe de Almeida ${ }^{3}$, Manoel Malheiros Tourinho ${ }^{4}$
}

\begin{abstract}
Resumo: O objetivo deste trabalho é identificar, descrever e analisar a literatura técnico-científica nacional sobre a estimativa de carbono em Sistemas Agroflorestais (SAFs), na região amazônica. Foi realizada uma revisão bibliográfica nas bases de dados dos portais eletrônicos mais significativos que tratam sobre o tema, postados entre os anos de 1998 e 2015. Conclui-se que, ainda há poucos estudos realizados sobre SAFs com fixação de carbono e são poucos os estudos apresentados sobre espécies nativas da Amazônia. Da mesma forma que apresentam poucas diferenças nas metodologias encontradas.
\end{abstract}

Palavras-chaves: Amazônia; Dióxido de carbono; SAF; Sustentabilidade.

\begin{abstract}
The objective of this work is to identify, describe and analyze the national scientific and technical publications to be carried on carbon fixation in Amazon Agroforestry Systems (AFS). We performed a list of online databases literature review of the most significant portal for digital collections of electronic papers that deal with the subject, posts between 1998 and 2012. It follows that there are few studies on AFS considering the carbon fixation and studies presented on Amazon rainforest plants. Therefore, also has been found few meaningful differences focused on Methodology in recent assessment articles.
\end{abstract}

Key words: Amazon rainforest; Carbon dioxide; AFS; Sustainability.

\footnotetext{
*Autor para correspondência

Recebido para publicação em 21/05/2015; aprovado em 10/10/2015

${ }^{1}$ Graduanda em Engenharia Florestal, UFRA, Belém-PA; 32462360, e-mail: irna.dias@ gmail.com.

${ }^{2}$ Graduanda em Engenharia Florestal, UFRA, e-mail: biacordeirocosta@ gmail.com

${ }^{3}$ Professor Doutor em Engenharia Elétrica, UFRA, e-mail: felipe.almeida@ufra.edu.br

${ }^{4}$ Professor Doutor em Sociologia Rural, UFRA, e-mail: paratourinho@gmail.com
} 


\section{INTRODUÇÃO}

Na Amazônia, os Sistemas Agroflorestais (SAFs) se apresentam como alternativa de agricultura sustentável, quando comparados à agricultura tradicional. Por definição, em um SAF, consorciam-se espécies florestais - tanto frutíferas como madeireiras - com cultivos agrícolas e, em algumas ocasiões, com animais. A partir deles, pode-se conciliar a segurança alimentar, o combate à pobreza rural e a conservação de recursos naturais. Também, vale dizer que os SAFs desempenham um papel sustentável na agroecologia, considerando-se, principalmente, a agricultura familiar (PALUDO, 2012). Desta maneira, os SAFs estão perfeitamente adequados na questão de restabelecer ouso correto da floresta e, de forma mais comprometida com as questões ambientais atuais, evitar os desequilíbrios ecológicos capazes de provocar mudanças climáticas e a intensificação do efeito estufa (FEARNSIDE, 2003). De fato, relacionado a $\mathrm{SAF}$, está, ainda, o controle na produção agroflorestal e na emissão/absorção de dióxido de carbono.

Devido ao uso da terra para agricultura, queima de combustíveis fósseis e desflorestamento, a concentração de dióxido de carbono (CO2) na atmosfera aumentou 42\%, desde a Revolução Industrial (TANHUA et al., 2015). Desta forma, o sequestro de carbono se tornou uma alternativa viável na captura de $\mathrm{CO} 2$ no controle da emissão de gases do efeito estufa e na redução dos efeitos do aquecimento global (ADUAN et al., 2003).

O Protocolo de Kyoto, assinado em 1997, fixou limite nas emissões de CO2 (C\&T BRASIL, 2006). Este documento passou a entrar em vigor em 2005 e seu texto deve ser substituído este ano por um novo acordo que será discutido na Conferência do Clima, em dezembro em Paris (COP21, 2015). Portanto, níveis de critérios são estabelecidos para diminuir emissões e, dessa forma, as nações desenvolvidas ocupam sua posição no círculo central e obrigam-se a fazer cortes em todos os setores de sua economia (UNITED NATIONS, 1998). Os emergentes, como Brasil, embora sejam grandes poluentes, podem participar do acordo, contudo, não estão obrigados a se comprometerem com metas, mas podem vender créditos de carbono, conforme o que foi assinado em Kyoto (GRÜTTER; KAPPEL e STAUB, 2002).

Dentre as possibilidades de metas, a inserção ecológica dos SAFs pode realizar a captura e fixação do carbono atmosférico por meio dos componentes do Sistema. Pois o ciclo dos SAFs é praticamente o mesmo do ciclo arbóreo, no qual a árvore é o elemento básico que influencia nos efeitos ambientais e propicia uma fixação eficaz de acordo com o arranjo espacial adotado no sistema. (SANCHEZ, 1995; FERNADES, 2004; SILVA, 2013). Nesse contexto, estão os benefícios da utilização de SAFs, tais que a redução do desmatamento e a viabilidade na captura e no controle da emissão de CO2, o que os tornam de valor sustentável.

Do ponto de vista acadêmico, avaliar a produção técnico-científica sobre SAF na Amazônia permite identificar áreas carentes de informações, ou agrupar saberes para consolidação de conhecimento (BRIENZA JÚNIOR et al., 2009). O presente estudo tem como objetivo fazer revisão bibliográfica da literatura técnico-científica de trabalhos que tratam sobre a estimativa de níveis de fixação de carbono em sistemas agroflorestais na região amazônica.

\section{MATERIAL E MÉTODOS}

Considera-se um estudo de revisão bibliográfica sobre a fixação do carbono em Sistemas Agroflorestais na Amazônia. Sua elaboração ocorreu pelo fato de se fazer um estudo capaz de verificar qual o andamento da pesquisa nacional, com relação a esse tema na literatura técnico-científica. Para isso, a busca bibliográfica foi realizada nas bases de dados do portal da CAPES, do INPA, da EMBRAPA, da Scielo e do PNAS (Proceedings of the National Academy of Sciences), nos idiomas inglês e português. Nessa pesquisa foram analisados aproximadamente 150 trabalhos, sendo, em torno de $60 \%$, publicações em anais de congressos. Nos artigos estudados foram incluídos artigos originais de revisão bibliográfica, estudos de caso e pesquisa que incluíssem a discussão sobre o assunto em questão.

\section{RESULTADOS E DISCUSSÃO}

De forma geral, visto que a floresta amazônica caracteriza-se como reservatório de carbono (NOBRE, 2001), deve-se considerar que em agroflorestas a renovação na captura de carbono é constante. Portanto, o incentivo à redução de $\mathrm{CO} 2$ na atmosfera cria expectativa para a divulgação do potencial florestal brasileiro, ainda assim, há escassez de publicações que possam demonstrar o potencial que os SAFs possuem para produção agrícola e recuperação de áreas degradadas (PAIXÃO, 2006; SILVA, 2013; KATO, 2012).

De acordo com a revisão bibliográfica apresentada em Torres et al, (2014), a Tabela 1 mostra os resultados da pesquisa original de Brancher (2010).

Tabela 1 - Descrição do estoque de carbono nos SAFs avaliados.

\begin{tabular}{|c|c|c|c|}
\hline SAFs & Espécies & Nome Vulgar & Estoque de Carbono Total \\
\hline SAF 1 & $\begin{array}{l}\text { Theobromacacao } \\
\text { Euterpe oleracea } \\
\text { Musa cavendishii } \\
\text { Hevea brasiliensis }\end{array}$ & $\begin{array}{l}\text { Cacau } \\
\text { Açaí } \\
\text { Banana } \\
\text { Seringa }\end{array}$ & $126,59 \mathrm{t} \mathrm{C} \mathrm{ha}^{-1}$ \\
\hline$\overline{\text { SAF } 2}$ & $\begin{array}{l}\text { Theobromacacao } \\
\text { Euterpe oleracea } \\
\text { Musa cavendishii } \\
\text { Hevea brasiliensis } \\
\text { Spondiasmombin } \\
\text { Schizolobiumamazonicum } \\
\text { Platymisciumtrinitatis }\end{array}$ & $\begin{array}{l}\text { Cacau } \\
\text { Açaí } \\
\text { Banana } \\
\text { Seringa } \\
\text { Taperebá } \\
\text { Paricá } \\
\text { Macacaúba }\end{array}$ & $128,41 \mathrm{t} \mathrm{C} \mathrm{ha}^{-1}$ \\
\hline
\end{tabular}




\begin{tabular}{|c|c|c|c|}
\hline \multicolumn{4}{|c|}{ Continuação Tabela 1} \\
\hline SAF 3 & $\begin{array}{l}\text { Theobromagrandiflorum } \\
\text { Euterpe oleracea } \\
\text { Tectonagrandis } \\
\text { Swieteniamacrophylla }\end{array}$ & $\begin{array}{l}\text { Cupuaçú } \\
\text { Açaí } \\
\text { Teca } \\
\text { Mogno }\end{array}$ & $122,80 \mathrm{t} \mathrm{C} \mathrm{ha}^{-1}$ \\
\hline SAF 4 & $\begin{array}{l}\text { Theobromagrandiflorum } \\
\text { Euterpe oleracea } \\
\text { Schizolobiumamazonicum }\end{array}$ & $\begin{array}{l}\text { Cupuaçú } \\
\text { Açaí } \\
\text { Paricá }\end{array}$ & $137,81 \mathrm{t} \mathrm{C} \mathrm{ha}^{-1}$ \\
\hline
\end{tabular}

A Tabela 1 apresenta a quantificação de carbono estocado nas parcelas de quatro tipos de SAFs, constituídos por plantas nativas e adaptadas ao bioma amazônico. Para a construção desses resultados, o autor utilizou dois métodos particularmente conhecidos na área de manejo e inventário florestal - para estimar o estoque de carbono em SAF'S. São eles: os métodos direto (ou destrutivo) e indireto (ou não destrutivo). O primeiro consiste na derrubada de árvores em parcelas fixas para posterior medição e pesagem do conteúdo (HIGUCHI, 1998). O segundo método utiliza dados de

Tabela 2 -Técnicas utilizadas para estimativa de CO2. inventário florestal, como as variáveis dendrométricas, entre elas, diâmetro à altura do peito (DAP), densidade média e volume da madeira, sendo o último a principal variável na obtenção de dados estatísticos. No método indireto, portanto, não há a necessidade de retirada de cobertura vegetal (HIGUCHI; CARVALHO, 1994; HIGUCHI, 1998).

Além dessas técnicas, também são utilizadas equações alométricas e métodos de cálculo e pesquisa, como demonstrados na Tabela 2, gerados a partir de pesquisas bibliográficas.

\begin{tabular}{ll}
\hline Referência & Método de cálculo e pesquisa
\end{tabular}

\begin{abstract}
Santos et al., $2004 \quad$ Estudo de caso envolvendo propriedades inventariadas em SAFs 50x50m. O método indireto, utilizado por meio de equações alométricas, estimou a altura total e o DAP $(\geq 5 \mathrm{~cm})$ para cálculo da média nos $7 \mathrm{SAFs}$, sendo $45 \%$ de carbono contido na espécie.
\end{abstract}

Bolfe et al., $2011 \quad$ Estudo de caso em 19 unidades com 3 parcelas cada, constituídas de área de $10 \mathrm{~m}^{2}$. A área inventariada, com o apoio de imagens de satélites, estimou a altura para DAP $>2,5 \mathrm{~cm}$. O cálculo de carbono utilizou 4 modelos alométricos para determinar a biomassa epígea, multiplicando posteriormente cada resultado por 0,485, conforme a técnica de Higuchi et al. (1998).

Souza et al., 2012
De acordo com a revisão bibliográfica apresentada na Tabela 2, os métodos e técnicas utilizados para obter estimativas de carbono são bastante similares e generalizados. Da mesma forma, todos os trabalhos baseiam-se em métodos que utilizam equações de recorrência, no que tange aos pressupostos na quantidade de biomassa carbônica.

Por isso, é importante que se utilizem métodos mais precisos que somatizem pesquisas envolvendo a absorção de CO2. Afinal, ainda há existência da margem de erro, associada às variáveis dendrométricas (NICOLETTI, 2011), ao erro na aplicabilidade de equações usadas de modo generalizado (NAIR et al., 2009) e à incerteza no emprego dos métodos (direto e indireto). Isto se deve ao fato de não haver consenso na elaboração de resultados, apesar da utilização do mesmo banco de dados (FEARNSIDE et al., 1993; HIGUCHI et al., 1994; FOSTER BROWN et al.1995).

\section{CONCLUSÕES}

A partir da revisão bibliográfica, foi identificada a carência de métodos mais sólidos e menos diversos para o estudo sobre a fixação de carbono em Sistemas Agroflorestais, implantados na região amazônica. Verificouse, ainda, que entre os trabalhos pesquisados, menos de $5 \%$ tratam desse assunto, especificamente. Portanto, o resultado ressalta a necessidade de mais estudos voltados para o desenvolvimento desse tipo de pesquisa, principalmente para as espécies nativas da Amazônia consorciadas no sistema.

\section{REFERÊNCIAS BIBLIOGRÁFICAS}

ADUAN, R. A.; VILELA, M. de F.; KLINK, C. A. Ciclagem de carbono em ecossistemas terrestres: o caso do Cerrado brasileiro. Ministério da agricultura, pecuária e 
abastecimento, documentos 105, ISS 1517 - 5111. EMBRAPA, 2003.

BRIENZA JÚNIOR, S.; MANESCHY, R. Q.; MOURÃO JÚNIOR, M.; GAZEL FILHO, A. B.; YARED, J. A. G.; GONÇALVES, D.; GAMA, M. B. Sistemas Agroflorestais na Amazônia Brasileira: Análise de 25 Anos de Pesquisas. Brazilian Jounal of Forestry Research. n. 60, 2009.

C\&T BRASIL. Entendendo a mudança do clima: um guia para iniciantes da Convenção-Quadro das Nações Unidas e seu Protocolo de Quioto. Disponível em www.mct.gov.br/clima. Acesso em 15 de abril de 2015.

COP 21. 21 a Conferência do clima em 2015. In: http://www.ambafrance-br.org/COP-21-Paris-2015.

FEARNSIDE, P. M. A floresta Amazônica nas mudanças globais. Manaus: INPA. 134 p. 2003.

FEARNSIDE, P. M.; FILHO, N. L.; FERNANDES, F. M. Rainforest Burning and the Global Budget: Biomass, Combustion, Efficiency, and Charcoal Formation in the Brazilian Amazon. J. of Geophysical Research, 98(D9): 16733-16743. 1993.

FOSTER BROWN, I.; MARTINELLI, L. A.; WAYT THOMAS, W.; MOREIRA, M. Z.; CID FERREIRA, C. A.; VIVTORIA, R. A. Uncertainty in the Biomass of Amazonian Forests: na Example from Rondônia, Brazil. Forest Ecology and Management, 75:175-189. 1995.

GRÜTTER, J., KAPPEL, R. e STAUB, P. The GHG market on the eve of Kyoto ratification. Nova Iorque: National Strategy Studies, 2002.

HIGUCHI, N.; SANTOS, J.; RIBEIRO, R. J.; MINETTE, L.; BIOT, Y. Biomassa da parte aérea da vegetação da floresta tropical úmida de terra firme da amazônia brasileira. Acta Amaz. V. 28, n. 2, 1998.

HIGUCHI, N.; CARVALHO JÚNIOR, J. A. Fitomassa e conteúdo de carbono de espécies arbóreas da Amazônia. In: Seminário Emissão x Sequestro de CO2 - Uma Nova Oportunidade de Negócios para o Brasil, 1994, Rio de Janeiro. Anais... Rio de Janeiro: CVRD, 1994. p. 125145.

KATO, O. R.; SHIMIZU, M. K.; BORGES, A. C. M. R.; AZEVEDO, C. M. B. C.; OLIVEIRA, J. S. L.; VASCONCELOS, S. S.; SÁ, T. D. A. Desenvolvimento da produção de frutas em sistemas agroflorestais no estado do Pará. XXII Congresso Brasileiro de Fruticultura. Bento Gonçalves, RS. 2012.

PALUDO, Rafael; COSTABEBER, José Antônio. Sistemas agroflorestais como estratégia de desenvolvimento rural em diferentes biomas brasileiros. Revista Brasileira de Agroecologia 7(2): 63-76 (2012) ISSN: 1980-9735
SILVA, I. C. Sistemas Agroflorestais: Conceitos e Métodos/ Ivan Crespo Silva. - 1. ed. - Itabuna: SBSAF, 2013.

TANHUA, T.; HANSSON, L.; ORR, J. C.; LORENZONI, L. Monitoring Ocean Carbon and Ocean Acidification. World Meteorological Organization Bulletin, v. 64, n. 1, 2015.

UNITED NATIONS. Kyoto protocol to the united nations framework convention on climate change, 1998. In: http://unfccc.int/resource/docs/convkp/kpeng.pdf

SILVA, M. R. G.; MARGARIDO, L. A. C.; COELHO, R. C. S.; APOLARI, J. P. Fixação de carbono por árvores nativas da floresta estacional semi-decidual plantadas em canavial no sistema de aleias: um estudo de caso. Revista Agrogeoambiental v4n2, agosto 2012.

BRANCHER, T. Estoque e ciclagem de carbono de sistemas agroflorestais em Tomé-Açu, Amazônia Oriental. 2010. 58 f. Dissertação (Mestrado em Ciências Ambientais) Universidade Federal do Pará, Belém, PA.

NAIR, P. K. R.; KUMAR, B. M.; NAIR, V. D. Agroforestry as a strategy for carbon sequestration. Review Article. J. Plant Nutr. SoilSci. 2009, 172, 10-23.

NICOLETTI, M. F. Comparação de métodos não-destrutivos de cubagem e árvores em pé visando à determinação da biomassa. Dissertação (Mestrado) - Escola Superior de Agricultura "Luiz de Queiroz". Piracicaba, 2011. 83 p: il.

PAIXÃO, F. A.; SOARES, C. P. B.; JACOVINE, L. A. G.; SILVA, M. L.; LEITE, H. C.; SILVA, G. F. Quantificação do estoque de carbono e avaliação econômica de alternativas em um plantio de eucalipto. R. Arvore, Viçosa-MG, v30, n.3, p.411-420, 2006

TORRES, C. M. M. E.; JACOVINE, L. A. G.; NETO, S. N. O.; BRIANEZI, D.; ALVES, E. B. B. M. Sistemas agroflorestais no Brasil: uma abordagem sobre a estocagem de carbono. Pesquisa Florestal Brasileira, Colombo, v. 34, n. 79, p. 235-244, jul./set. 2014. 